\title{
Desenvolvimento e Aplicação de um Protocolo Fisioterapêutico em Pacientes com Mastalgia
}

\section{Development and Application of a Physiotherapeutic Protocol in Patients with Mastalgia}

\author{
Rayssa de Cássia Ramos Nascimento ${ }^{1}$ \\ Layra Estelita Souza da Luz ${ }^{2}$ \\ Pedro Renan Nascimento Barbosa ${ }^{3}$ \\ Wanessa Carvalho Wanzeler ${ }^{4}$ \\ Denise da Silva Pinto 5 \\ Cibele Nazaré Câmara Rodrigues ${ }^{6}$
}

\section{RESUMO}

Objetivo: Verificar a influência do protocolo fisioterapêutico, composto por eletroterapia e exercício físico em mulheres com mastalgia. Metodologia: Trata-se de um estudo clínico não-controlado com amostra intencional de dez mulheres com mastalgia, aprovado pelo Comitê de Ética em Pesquisa (CEP) e realizado no Centro de Atenção a Saúde da Mulher e da Criança (CASMUC) da Universidade Federal do Pará (UFPA). Os instrumentos avaliativos aplicados antes e após o protocolo terapêutico foram à versão brasileira do Questionário de Qualidade de Vida Short Form-36 (SF-36) e Escala Visual Analógica (EVA), analisados pelo teste $t$ Student, e Escala Medical Research Council (MRC), analisado pelo teste de Wilcoxon. O protocolo da pesquisa consistiu nas pacientes serem submetidas às sessões terapêuticas, duas vezes semanais, totalizando dez sessões. As condutas foram compostas pela utilização da neuroestimulação elétrica transcutânea (TENS) associado ao exercício físico. Resultados: Ocorreu redução significativa da dor na mama direita $\left(p=0,0117^{\star}\right)$ e esquerda $\left(p=0,0464^{*}\right)$, pela EVA, melhora significativa na análise individual dos domínios de qualidade de vida relacionada à dor $\left(p=0,0380^{*}\right)$ e saúde mental $\left(p=0,0015^{\star}\right)$ pelo SF-36 e melhora relativa da força muscular pelo MRC. Conclusão: O protocolo fisioterapêutico demonstrou eficácia frente ao quadro álgico das participantes, assim como resultados positivos na qualidade de vida e força muscular.

\section{DESCRITORES}

Mastalgia. Eletroterapia. Exercício Físico.

\begin{abstract}
Objective: Verify the influence of the physiotherapeutic protocol consisting of electrotherapy and physical exercise in women with mastalgia. Methodology: This was an uncontrolled clinical trial with an intentional sample of ten women with mastalgia, approved by the Research Ethics Committee and carried out at the Center for Attention to the Health of Women and Children of the Federal University of Pará. The evaluation instruments applied before and after the therapeutic protocol were the Brazilian version of the Short Form-36 Quality of Life Questionnaire (SF-36) and Visual Analogue Scale (VAS), which was analyzed by the Student's t-test, as well as the Medical Research Council (MRC) scale, which was analyzed by the Wilcoxon's test. The research protocol consisted of patients submitted to therapeutic sessions, twice a week, totaling ten sessions, the conducts were composed using the Transcutaneous Electrical Neurostimulation (TENS) associated with physical exercise. Results: There was a significant reduction in the AVS of pain in both the right $\left(p=0.0117^{*}\right)$ and left breasts $\left(p=0.0464^{*}\right)$, significant improvement on each domain of quality of life related to pain $\left(p=0.0380^{*}\right)$ and mental health $\left(p=0.0015^{*}\right)$ by the SF-36 and relative improvement of muscle strength by the MRC. Conclusion: The physiotherapeutic protocol demonstrated effectiveness in view of the participants' pain, as well as positive results in quality of life and muscle strength.
\end{abstract}

\section{DESCRIPTORS}

Mastalgia. Electrotherapy. Physical Exercise.

${ }^{1}$ Fisioterapeuta residente em Intensivismo do Hospital Universitário Regional dos Campos Gerais, Universidade Estadual de Ponta Grossa, Ponta Grossa, PR, Brasil;

${ }^{2}$ Fisioterapeuta graduada pela Universidade Federal do Pará (UFPA);

${ }^{3}$ Fisioterapeuta residente em Saúde da mulher e da criança pelo Hospital Santo Antônio Maria Zaccaria, Universidade Federal do Pará, Bragança, PA, Brasil;

${ }^{4}$ Graduanda em Fisioterapia pela Universidade Federal do Pará (UFPA);

${ }^{5}$ Docente da Faculdade de Fisioterapia e Terapia Ocupacional da Universidade Federal do Pará (UFPA);

${ }^{6}$ Doutora em teoria e pesquisa do comportamento humano pela Universidade Federal do Pará (UFPA). 
A mastalgia, também chamada de mastodinia ou dor mamária, é a dor unilateral ou bilateral nas mamas que varia de tensão leve até agonizante e apresenta-se de forma isolada ou irradiada para a parede torácica e membros superiores ${ }^{1-3}$. Ainda não há estudos que abordem a prevalência global ou continental da mastalgia, havendo dados apenas de pequenas amostras em populações aleatórias ${ }^{4-6}$.

A dor mamária se classifica em cíclica, aclíclica e extramamária. A cíclica, tipo mais comum de dor mamária, está relacionada à menstruação, apresentando-se bilateralmente. A aclíclica é a dor na mama ou na parede torácica não relacionada ao período menstrual, sendo na maioria das vezes unilateral $^{2,7}$. Já o tipo extramamária se refere à dor originada em outras áreas além da mama, com origem musculoesquelética, cardíaca, gastrointestinal, além de patologias neurológicas ou de origem psicológica ${ }^{3,7}$.

A mastodinia é mais comum na faixa etária dos 30 aos 50 anos e seu prognóstico varia de acordo com a classificação e tipo de tratamento empregado². No Brasil, de acordo com a Sociedade Brasileira de Mastologia, cerca de até $70 \%$ da população feminina apresenta ou manifestará algum tipo de dor ou desconforto nas mamas ${ }^{8}$, podendo afetar assim diversas dimensões da saúde e bem-estar das mulheres, interferindo nas atividades sociais, físicas, sexuais e relacionadas ao trabalho ${ }^{5}$.

Acerca dos tratamentos utilizados para o alívio da dor mamária, na literatura encontram-se o uso de anti-inflamatórios ${ }^{9} \mathrm{com}$ grande eficácia, aconselhamento psicológico ${ }^{3}$, terapia não hormonal ${ }^{10}$, terapia hormonal ${ }^{11} \mathrm{e}$ cirurgia em alguns casos ${ }^{12}$.
A partir disso a fisioterapia, em quadro de algia, é uma alternativa de tratamento não farmacológico para alívio da dor e melhora da qualidade de vida, mediante a utilização de recursos eletroterapêuticos, como a Neuroestimulação Elétrica Transcutânea (TENS) ${ }^{13}$, e exercício físico ${ }^{10}$. A relevância clínica deste estudo se dá pela escassez de registros na literatura científica de um protocolo fisioterapêutico com tais abordagens.

Por meio dos achados na literatura a dor mamária demonstra ser um problema de saúde pública ${ }^{5}$ recorrente entre as mulheres, a hipótese construída foi que a fisioterapia com a utilização de eletroterapia e cinesioterapia pode gerar desfechos positivos para indivíduos com estas queixas, favorecendo o índice de força muscular e qualidade de vida. Diante disso, o objetivo desta pesquisa é verificar a influência de um protocolo fisioterapêutico na mastalgia.

\section{METODOLOGIA}

Trata-se de um estudo clínico não controlado, com amostra intencional selecionada por conveniência entre mulheres que frequentavam o serviço médico do Centro de Atendimento à Saúde da Mulher e da Criança (CASMUC) da Universidade Federal do Pará (UFPA). A pesquisa cumpriu com os princípios éticos da Declaração de Helsinque (2000) e foi apresentado ao Comitê de Ética em Pesquisa envolvendo seres humanos do Instituto de Ciências da Saúde da UFPA (ICS-UFPA) pelo CAAE 92229718.9.0000.0018., e aprovado sob parecer de número 2.929.131.

Foram incluídas 10 mulheres maiores de 18 anos, com queixa primária de dor ma- 
mária que não houvesse associação com câncer de mama. Foram excluídas da pesquisa mulheres com hipertensão arterial sistêmica não controlada, disfunções cognitivas e/ou submetidas a procedimento cirúrgico como a mastectomia.

Foi realizada uma avaliação inicial (AV1) e final (AV2), por um único avaliador, no qual foram registrados os dados gerais e específicos, dentre eles os antecedentes pessoais e familiares, queixa principal, história da doença atual, histórico ginecológico, obstétrico e mamário, a versão Brasileira do Questionário de Qualidade de Vida Short Form-36 (SF-36) ${ }^{14}$, Escala Visual Analógica $(E V A)^{15}$ e Escala Medical Research Council $(\mathrm{MRC})^{16}$.

Após $A V 1$, as pacientes foram submetidas às sessões terapêuticas, duas vezes semanais, com duração de uma hora, totalizando dez sessões. Antes de iniciar a sessão, posicionavam-se as sujeitas em sedestação, aguardando por cinco minutos para a aferição da pressão arterial (PA), sendo o atendimento realizado apenas com valores dentro da normalidade ${ }^{17}$.

Posteriormente, aplicava-se a TENS, recurso eletroterapêutico utilizado com intuito de redução dos estímulos dolorosos por meio da neuromodulação, na modalidade graduada para dor crônica, com duração de 30 minutos, frequência de $5 \mathrm{~Hz}$ e largura de pulso de $200 \mu \mathrm{s}^{18}$. O posicionamento das pacientes foram em decúbito dorsal com os eletrodos posicionados nos quadrantes das mamas.

Em bipedestação, foram realizados alongamentos para grandes grupos musculares de membros superiores, sendo mantidos por 20 segundos, de forma ativo-assistida ou ativo-livre. Em seguida, foram realizados exercícios para musculatura flexora, extensora, abdutora, adutora e rotadores internos e externos da articulação do ombro. Utilizaram-se recursos como halter, faixa elástica, bastão e caneleira para potencializar os exercícios. O volume da atividade variava de acordo com a capacidade da paciente, evoluindo com carga ou com número de repetições. Ao final do atendimento a PA era aferida para liberação terapêutica sem intercorrências.

Análise estatística

Os resultados obtidos nos testes e escalas aplicadas foram analisados quanto à diferença entre AV1 e AV2, e para averiguação de significância estatística, foi utilizado o software BioEstat 5.0, nas variáveis EVA e SF-36 utilizou o Teste $t$ Student para amostras pareadas e Wilcoxon na comparação da força muscular pelo MRC, considerando a normalidade dos dados. Foram considerados estatisticamente significantes resultados com $p \leq 0,05$.

\section{RESULTADOS}

O objetivo desta pesquisa foi verificar a influência de um protocolo de eletroterapia associado ao exercício físico na mastalgia. Este é o primeiro estudo que avalia o impacto da aplicação destes recursos sobre o quadro de dor, qualidade de vida e força muscular nesta população. A partir dos dados coletados, foram descritos na tabela 1 as características da amostra, na qual detalham aspectos epi- 
Tabela 1. Aspectos epidemiológicos e clínicos das participantes do estudo

\begin{tabular}{|c|c|c|c|c|c|}
\hline $\begin{array}{c}\text { Aspectos } \\
\text { Epidemiológicos }\end{array}$ & $n^{\circ}$ Absoluto & $\%$ & Aspectos Clínicos & $\mathrm{n}^{\circ}$ Absoluto & $\%$ \\
\hline \multicolumn{3}{|c|}{ Idade } & \multicolumn{3}{|c|}{ Gestação } \\
\hline $18-59$ & 06 & 60 & Sim & 09 & 90 \\
\hline \multicolumn{3}{|c|}{ Etnia } & \multicolumn{3}{|c|}{ Menopausa } \\
\hline Negra & 02 & 20 & $\leq 50$ anos & 05 & 50 \\
\hline Parda & 06 & 60 & $>50$ anos & 004 & 40 \\
\hline Branca & 02 & 20 & Não se aplica & 1 & 10 \\
\hline \multicolumn{3}{|c|}{ Estado Civil } & \multicolumn{3}{|c|}{ Menarca } \\
\hline Casadas & 03 & 30 & $\leq 12$ anos & 02 & 20 \\
\hline Viúvas & 03 & 30 & $13-14$ anos & 06 & 60 \\
\hline Solteiras & 04 & 40 & $\geq 15$ & 02 & 20 \\
\hline \multicolumn{3}{|c|}{ Escolaridade } & \multicolumn{3}{|c|}{ Uso de Anticoncepcional } \\
\hline $1^{\circ}$ grau incompleto & 03 & 30 & Sim & 08 & 80 \\
\hline $1^{\circ} \mathrm{grau}$ completo & 02 & 20 & Não & 02 & 20 \\
\hline $2^{\circ}$ grau incompleto & 01 & 10 & \multicolumn{3}{|c|}{ Cirurgia da Mama } \\
\hline $2^{\circ}$ grau completo & 04 & 40 & Sim & 02 & 20 \\
\hline & IC & & Não & 08 & 80 \\
\hline Normal & 05 & 50 & \multicolumn{3}{|c|}{ Nódulos Benignos } \\
\hline Sobrepeso & 03 & 30 & Sim & 01 & 10 \\
\hline Obesidade & 02 & 20 & Não & 09 & 90 \\
\hline
\end{tabular}

demiológicos e clínicos.

Os resultados obtidos na comparação da EVA entre as avaliações, demonstram que houve diminuição significativa de dor mamária na mama direita (MD - $p=0,0117^{*}$ ) e mama esquerda ( $\left.M E-p=0,0464^{*}\right)$. Os valores exatos de média, desvio padrão e $p$ valor encontram-se descritos na tabela 2.

De acordo com a análise dos dados da qualidade de vida pelo SF-36, o escore total não apresentou valor de significância $(p=0,3232)$. Entretanto, na análise individual, os domínios que obtiveram significância após intervenção correspondem à qualidade de vida relacionada à dor $\left(p=0,0380^{*}\right)$ e saúde mental $\left(p=0,0015^{*}\right)$.

Neste estudo, os domínios de capaci- dade funcional, aspectos físicos, estado geral de saúde e aspectos sociais apresentaram aumento relativo nos valores de acordo com a média, porém não significativos, enquanto vitalidade e aspectos emocionais não apresentaram aumento relativo e significância na análise estatística. Os valores de média, desvio padrão e $p$ valor encontram-se descritos na tabela 3.

A partir da análise dos dados da força muscular pelo MRC, houve aumento dos graus de força pelas médias apresentadas na maioria dos grupos musculares presentes na articulação, porém não significativos estatisticamente. Os valores de média, desvio padrão e $p$ valor encontram-se descritos na tabela 4 . 
Tabela 2. Valores obtidos antes e após da EVA

EVA

\begin{tabular}{|c|c|c|c|c|c}
\hline \multicolumn{2}{|c|}{$\mathrm{MD}$} & \multirow{2}{*}{$p$} & \multicolumn{2}{|c|}{$\mathrm{ME}$} & \multirow{2}{*}{$p$} \\
\cline { 1 - 1 } $\mathrm{AV} 1$ & $\mathrm{AV} 2$ & & $\mathrm{AV} 1$ & $\mathrm{AV} 2$ & $0,0464^{*}$ \\
\hline $5 \pm 3$ & $2 \pm 2$ & $0,09117^{*}$ & $4 \pm 3$ & $1,7 \pm 2$ & 0 \\
\hline
\end{tabular}

Os valores apresentados são referentes a média e desvio padrão da Escala Visual Analógica de Dor (EVA); MD: mama direita; ME: mama esquerda; AV1: avaliação inicial; AV2: avaliação final; * Teste $t$ student, considerando significativo $p \leq 0,05$. Os valores apresentados são referentes a média e desvio padrão da Escala Visual Analógica de Dor (EVA); MD: mama direita; ME: mama esquerda; AV1: avaliação inicial; AV2: avaliação final; * Teste $t$ student, considerando significativo $p \leq 0,05$.

Tabela 3. Valores obtidos antes e após análise do SF-36

\begin{tabular}{|c|c|c|c|c|c|}
\hline \multirow{2}{*}{ SF-36 } & \multicolumn{2}{|c|}{ Média $\pm \mathrm{DP}$} & \multicolumn{2}{|c|}{ Mín. - Máx. } & \multirow{2}{*}{$P$} \\
\hline & AV1 & AV2 & $\mathrm{AV} 1$ & AV2 & \\
\hline Escore Total & $97,5 \pm 10,3$ & $100,4 \pm 13,6$ & $80,0-113,5$ & $79,1-118,4$ & 0,3232 \\
\hline Capacidade funcional & $59,5 \pm 23,5$ & $58,5 \pm 22,7$ & $30-95$ & $30-95$ & 0,8309 \\
\hline Aspecto físico & $44,9 \pm 42,2$ & $55,0 \pm 38,7$ & $0-100$ & $0-100$ & 0,5522 \\
\hline Dor & $42,2 \pm 16,75$ & $59,7 \pm 22,14$ & $21-74$ & $21-90$ & $0,0380^{*}$ \\
\hline Estado geral de saúde & $47,1 \pm 12,2$ & $49,5 \pm 14,1$ & $30-72$ & $35-72$ & 0,3952 \\
\hline $\begin{array}{l}\text { Vitalidade } \\
\text { Aspecto social }\end{array}$ & $\begin{array}{l}62,0 \pm 19,3 \\
74,8 \pm 20,4\end{array}$ & $\begin{array}{l}61,0 \pm 22,2 \\
74,9 \pm 22,8\end{array}$ & $\begin{array}{c}30-85 \\
50-100\end{array}$ & $\begin{array}{l}10-80,0 \\
50-100\end{array}$ & $\begin{array}{l}0,7895 \\
0,9756\end{array}$ \\
\hline Aspecto emocional & $43,3 \pm 44,6$ & $60,0 \pm 46,6$ & $0-100$ & $0-100$ & 0,3800 . \\
\hline Saúde mental & $67,6 \pm 25,4$ & $75,6 \pm 23,9$ & $16-96$ & $32-100$ & $0,0015^{*}$ \\
\hline
\end{tabular}

SF - 36: Questionário de qualidade de vida; DP: Desvio padrão; MíN.: valor mínimo; MÁX: valor máximo; AV1: avaliação inicial; AV2: avaliação final; * Teste T de student, considerando significativo $p \leq 0,05$.

Tabela 4. Valores obtidos antes e após aplicação da MTC

\begin{tabular}{l|c|c|c|c|c|c}
\hline \multirow{2}{*}{ MRC } & \multicolumn{2}{c|}{ MSD } & & \multicolumn{2}{c}{ MSE } & \multirow{2}{*}{$P$} \\
\cline { 2 - 3 } \cline { 5 - 7 } & AV1 & AV2 & $P$ & AV1 & AV2 & \\
\hline Flexores & $4,2 \pm 0,7$ & $4,7 \pm 0,4$ & 0,0579 & $4,1 \pm 0,8$ & $4,1 \pm 0,7$ & 0,5000 \\
Extensores & $4,3 \pm 0,8$ & $4,6 \pm 0,5$ & 0,0544 & $4,3 \pm 0,8$ & $4,6 \pm 0,6$ & 0,5440 \\
Abdutores & $4,0 \pm 0,8$ & $4,3 \pm 0,8$ & 0,1367 & $4,0 \pm 0,2$ & $4,2 \pm 0,2$ & 0,2113 \\
Adutores & $4,3 \pm 0,6$ & $4,4 \pm 0,5$ & 0,3429 & $4,3 \pm 0,6$ & $4,3 \pm 0,4$ & 0,5000 \\
Rotadores Internos & $4,7 \pm 0,6$ & $4,7 \pm 0,4$ & 0,5000 & $4,9 \pm 0,3$ & $4,6 \pm 0,5$ & 0,0544 \\
Rotadores Externos & $4,5 \pm 0,7$ & $4,6 \pm 0,5$ & 0,2965 & $4,7 \pm 0,4$ & $4,5 \pm 0,5$ & 0,1807 \\
\hline
\end{tabular}




\section{DISCUSSÃO}

A partir dos resultados obtidos, a significância na EVA evidenciou a eficácia do protocolo frente ao quadro álgico das pacientes. Resultados semelhantes foram observados em um ensaio clínico randomizado e controlado ${ }^{10}$ que buscou avaliar os efeitos do exercício físico em 20 mulheres com queixas de mastalgia, por meio de métodos para quantificar a dor e questionário de qualidade de vida SF-36. Após o programa de exercício, realizado três vezes semanais durante um período de seis semanas, o grupo exercício obteve melhora significativa sobre o grupo controle no componente sensitivo do questionário de dor $\left(p=0,012^{*}\right)$ e nos valores da escala visual analógica $\left(p=0,016^{*}\right)$.

Uma revisão sistemática abordou o manejo clínico para mastalgia em um período de quarenta anos (1975 a 2015) e demonstrou que o tratamento de primeira linha são orientações sobre posicionamento do sutiã e, em segunda linha, uso de terapias medicamentosas com equilíbrio e cautela, sendo os anti-inflamatórios tópicos não esteroidais e o Ormeloxifene os de melhores resultados clínicos com menores efeitos colaterais ${ }^{9}$. Outras medidas de tratamento também foram propostas como terapias naturais e conservadoras (Danazol), tratamentos endócrinos (Zoladex) tratamentos alternativos (acupuntura, relaxamento, cinesiologia aplicada e terapia administrada via audiocassettes) e cirurgias, porém tendo alívio sintomático limitado sobre a dor. Uso de vitaminas ${ }^{19,20}$, fitoterápicos ${ }^{21,22}$ e terapia nutricional também são utilizados como terapêutica ${ }^{23}$.

Em relação a cinesioterapia, um estudo de revisão, realizado com pacientes com dor crônica, demonstrou que o exercício físico de cunho aeróbico, força, flexibilidade, amplitude de movimento e programas de treinamento básicos ou de equilíbrio, produz efeitos favoráveis na redução da intensidade da dor, melhora da função física e psicológica, culminando em melhor qualidade de vida ${ }^{24}$.

Um estudo de revisão sistemática com metanálise avaliou o desfecho da aplicação da TENS e da Corrente Interferencial sobre alívio da dor aguda e crônica, com resultados significativos e similares na diminuição da dor ${ }^{25}$. Do mesmo modo, uma revisão sistemática abordou o efeito da TENS na dor crônica, os achados foram positivos em relação ao uso de medicamentos analgesicos, contudo não foi possível concluir com segurança a efetividade desta abordagem devido ao baixo nível de evidência científica dos estudos e limitação no número de participantes ${ }^{26}$.

Um estudo transversal comparou 40 mulheres com mastalgia e 40 mulheres saudáveis para avaliar a qualidade de vida pelo SF-36, índices de depressão pela Escala de Depressão de Hamilton e ansiedade pela Escala de Ansiedade de Hamilton, observando que em mulheres com mastalgia a qualidade de vida era inferior em todas as subcategorias e nos domínios de função física $\left(p=0,04^{*}\right)$, dor $\left(p=0,02^{*}\right)$, saúde geral $\left(p=0,03^{*}\right)$, e vitalidade $\left(p=0,008^{*}\right)$ foram significativamente baixos ${ }^{27}$.

Os resultados desta pesquisa demonstram melhora em todos os domínios do SF-36, com significância nos itens relacionados à dor $\left(p=0,0380^{*}\right)$ e saúde mental $\left(p=0,0015^{\star}\right)$. Achados semelhantes ao desta pesquisa foram encontrados em um ensaio clínico randomizado controlado, no qual utilizou-se da balneoterapia em pacientes com mastalgia, com desfecho positivo e significativo na avaliação 
do SF-36 em cinco domínios, entre eles dor e saúde mental ${ }^{28}$. Ademais um ensaio clínico controlado avaliou a influência do exercício sobre a dor e a qualidade de vida em mulheres com mastalgia, os resultados do SF-36 para o grupo experimental obtiveram significância nos domínios de dor, aspecto físico e social ${ }^{10}$.

Outros tratamentos também foram abordados em uma revisão sistemática, buscando verificar terapêuticas voltadas para qualidade de vida, redução da dor e efeitos colaterais dessa população. A amostra total de 2.100 pacientes, coletados dos vinte e três estudos incluídos, demonstrou que medicamentos, como Danazol e Bromocriptina, e multivitaminas favorecem uma melhor qualidade de vida no âmbito sexual, rotina diária, humor e sono ${ }^{3}$.

Após a AV2, houve aumento relativo dos graus de força, porém não significativos. Achados em uma revisão sistemática condizem com tais resultados onde explanam que o fortalecimento associado aos alongamentos de estabilizadores da cintura escapular favorece o aumento da funcionalidade do ombro associada à redução da dor, sendo assim uma opção no tratamento conservador de tais pacientes ${ }^{29}$.

Outro estudo, com protocolo de exercício semelhante ao da pesquisa atual, buscou avaliar os efeitos da fisioterapia sobre a qualidade de vida e o quadro clínico de 10 pacientes com câncer de mama, avaliando força e flexibilidade. A força muscular foi mensurada pelo teste de uma repetição máxima para exercícios de flexão, extensão e abdução de ombro, sendo o protocolo composto de alongamentos e exercício de resistência para membros superiores, variando em carga e intensidade de acordo com as semanas. O resultado demonstrou significância nos valores de força muscular em membros superiores após a intervenção para movimento de flexão, extensão e abdução $\left(p<0,01^{*}\right)^{30}$.

Limitações do estudo

Ressaltamos a presença de limitações importantes quanto ao tamanho da amostra do estudo, que pode ser explicado por fatores de vulnerabilidade econômica, aspectos geográficos, como acesso ao centro de atendimento, e aspectos pessoais não relatados que influenciaram na frequência das sessões terapêuticas.

\section{CONCLUSÃO}

O protocolo proposto para o tratamento de mastalgia, contendo eletroterapia e exercícios físicos, demonstrou eficácia frente ao quadro álgico, bem como resultados positivos em domínios da qualidade de vida relacionada à dor e saúde mental, além do aumento ou manutenção da força muscular.

\section{REFERÊNCIAS}

1. Marinov B, Andreeva A, Pandurska A. [Mastodynia Premenstrual syndrome]. AkushGinekol (Sofiia). 2014 53(6):36-40.

2. Bundred NJ. Breast pain. BMJ ClinEvid. 2007; 04:812.

3. Groen JW, Grosfeld S, Wilschut JA, Bramer WM, Ernst

MF, Mullender MM. Cyclic and non-cyclic breast-pain: A systematic review on pain reduction, side effects, and quality of life for various treatments. Eur $\mathrm{J}$ ObstetGynecolReprod Biol. 2017; 219:74-93.

4. Koçoğlu D, Kurşun S, Akın B, Altuntug K. Mastalgia and 
associated factors: a cross-sectional study. Agri. 2017; 29(3):100-108.

5. Brown N, White J, Brasher A, Scurr J. The experience of breast pain (mastalgia) in female runners of the 2012 London Marathon and its effect on exercise behaviour. $\mathrm{Br}$ J Sports Med. 2014; 48(4):320-325.

6. Burbage J, Cameron L. An investigation into the prevalence and impact of breast pain, bra issues and breast size on female horse riders. J Sports Sci. 2017; 35(11):10911097.

7. Chase C, Wells J, Eley S. Caffeine and Breast Pain: Revisiting the Connection. Nursing for Women'sHealth. $2011 ; 15(4): 286-294$.

8. Dores mamárias podem ser confundidas com câncer de mama Sociedade Brasileira de Mastologia. 2018).

9. Hafiz SP, Barnes NLP, Kirwan CC. Clinical management of idiopathic mastalgia: a systematic review. J Prim Health Care. 2018; 10(4):312-323.

10. Genç A, Çelebi MM, Çelik SU, Atman ED, Kocaay AF, Zergeroğlu AM, et al. The effects of exercise on mastalgia. Phys Sportsmed. 2017; 45(1):17-21.

11. Files JA, Miller VM, Cha SS, Pruthi S. Effects of different hormone therapies on breast pain in recently postmenopausal women: findings from the Mayo Clinic KEEPS breast pain ancillary study. J Womens Health (Larchmt). 2014; 23(10):801-805.

12. Kataria K, Dhar A, Srivastava A, Kumar S, Goyal A. A systematic review of current understanding and management of mastalgia. Indian J Surg. 2014; 76 (3):217-222.

13. Lozier CC, Nugent SM, Smith NX, Yarborough BJ, Dobscha SK, Deyo RA, et al. Correlates of Use and Perceived Effectiveness of Non-pharmacologic Strategies for Chronic Pain Among Patients Prescribed Long-term Opioid Therapy. J Genlntern Med. 2018; 33(1):46-53.

14. Ciconelli RM. Tradução para o português e validação do questionário genérico de avaliação de qualidade de vida "Medical OutcomesStudy 36-item Short-Form Health Survery (SF-36)". São Paulo. 1997;1-148

15. Cunha AC, Burke TN, França FJ, Marques AP. Effect of global posture reeducation and of static stretching on pain, range of motion, and quality of life in women with chronic neck pain: a randomized clinical trial. Clinics (Sao Paulo). 2008; 63(6):763-770.

16. Sullivan SB, Schmitz TJ, Fulk GD. Fisioterapia: Avaliação e tratamento. 6. ed. São Paulo: Manole, 2017.

17. V Diretrizes de Monitorização Ambulatorial da Pressão Arterial (MAPA) e III diretrizes de monitorização residencial da pressão arterial (MRPA). ArqBrasCardiol. 2011; 97(3):07-17.

18. Agne JE. Eletrotermofototerapia. 2. ed.Santa Maria, 2013.

19. Hajizadeh $\mathrm{K}$, AlizadehCharandabi SM, Hasanzade R, Mirghafourvand M. Effect of vitamin E on severity and duration of cyclic mastalgia: A systematic review and meta-analysis. Complement Ther Med. 2019; 44:1-8.

20. Shobeiri F, Oshvandi K, Nazari M. Clinical effectiveness of vitamin $E$ and vitamin B6 for improving pain severity in cyclic mastalgia. Iran J Nurs Midwifery Res. 2015; 20(6):723-727.

21. Saghafi N, Rhkhshandeh $\mathrm{H}$, Pourmoghadam N, Pourali L, Ghazanfarpour M, Behrooznia A, et al. Effectiveness of Matricariachamomilla (chamomile) extract on pain contro of cyclic mastalgia: a double-blind randomised controlled trial. J ObstetGynaecol. 2018; 38(1):81-84.

22. Huseini HF, Kianbakht S, Mirshamsi MH, Zarch AB. Effectiveness of Topical Nigella sativa Seed Oil in the Treatment of Cyclic Mastalgia: A Randomized, Triple-Blind, Active, and Placebo-Controlled Clinical Trial. Planta Med. 2016; 82(4):285-288

23. Mansel RE, Das T, Baggs GE, Noss MJ, Jennings WP Cohen J, et al. A Randomized Controlled Multicenter Tria of an Investigational Liquid Nutritional Formula in Women with Cyclic Breast Pain Associated with Fibrocystic Breast Changes. J Womens Health (Larchmt). 2018; 27(3):333340.

24. Geneen LJ, Moore RA, Clarke C, Martin D, Colvin LA Smith BH. Physical activity and exercise for chronic pain in adults: an overview of Cochrane Reviews. Cochrane Database Syst Rev. 2017; 24(4):1-76.

25. Almeida CC, Silva VZMD, Júnior GC, Liebano RE, Durigan JLQ. Transcutaneous electrical nerve stimulation and interferential current demonstrate similar effects in relieving acute and chronic pain: a systematic review with meta-analysis. Braz J Phys Ther. 2018; 22(5):347-354.

26. Gibson W, Wand BM, Meads C, Catley MJ, O'Connel NE. Transcutaneous electrical nerve stimulation (TENS) for chronic pain - an overview of Cochrane Reviews. Cochrane Database Syst Rev. 2019; 3(4):1-31.

27. Kanat BH, Atmaca M, Girgin M, Ilhan YS, Bozdağ A, Özkan $Z$, et al. Effects of Mastalgia in Young Women on Quality of Life, Depression, and Anxiety Levels. Indian J Surg. 2016; 78(2):96-9.

28. Genç A, Çelik SU, Evcik D, Atman ED, Elhane AH, Genç V. Balneotherapy is an alternative treatment for mastalgia: a randomized controlled trial. European Journal of Integrative Medicine. 2018; 19:100-104.

29. Stapait EL, Dalsoglio M, Ehlers AM, Santos GM. Fortalecimento dos estabilizadores da cinturaescapular na dor no ombro: revisão sistemática. Fisioter. Mov. 2013; 26(3):667-675.

30. Leites GT, Knorst MR, Lima CHLd, Zerwes FP, Frison VB. Fisioterapia em oncologia mamária: qualidade de vida e evolução clínico funcional.CienSaude Colet. 2010; 3(1):14-21.

\section{CORRESPONDÊNCIA}

Rayssa de Cássia Ramos Nascimento

Av. General Carlos Cavalcanti, 5775,

Condomínio Universiflat plus, bloco Elitt, apto 1,

CEP: 84025-000, Ponta-Grossa/PR, Brasil.

Email: rayssaramosnascimento@gmail.com 